\title{
On relative performance, remuneration and risk taking of asset managers
}

\author{
Emilio Barucci • Gaetano La Bua . \\ Daniele Marazzina
}

Received: date / Accepted: date

\begin{abstract}
We analyze the asset management problem when the manager is remunerated through a scheme based on the performance of the fund with respect to a benchmark and his/her choices are driven by a power utility function. We show that it is not the asymmetric-fulcrum type feature of the scheme that makes the difference in preventing excessive risk taking in case of a poor performance. To prevent gambling when the performance deteriorates, it is important not to provide a fixed fee to the asset manager, and that remuneration is sensitive to a very poor relative performance as in the case of a capital stake or of a management fee with flow funds. We provide empirical evidence on the mutual fund industry showing excessive risk taking in case of a very poor performance and limited risk taking in case of overperformance with respect to the benchmark. These results agree with a remuneration scheme including a fixed fee and a cap.
\end{abstract}

Keywords relative performance $\cdot$ remuneration $\cdot$ asset manager $\cdot$ portfolio

JEL classification G11 - G23 - G32

Acknowledgements We thank an anonymous referee for useful comments, the usual disclaimers apply.

\section{Introduction}

The performance of a fund with respect to a benchmark (relative performance) affects the asset management through two different channels: the

E. Barucci, G. La Bua, D. Marazzina

Department of Mathematics, Politecnico di Milano.

Piazza Leonardo da Vinci, I-20133 Milano (Italy)

Tel.: +39 0223994630 E-mail: emilio.barucci@polimi.it, gaetano.labua@polimi.it, daniele.marazzina@polimi.it 
remuneration scheme of the manager, and the flow of funds which is typically a convex increasing function of the relative performance of the fund, see [Sirri and Tufano(1998), Chevalier and Ellison(1997)]. In this paper we investigate how remuneration schemes based on the relative performance affect the asset management of the fund providing incentives to take risk in excess with respect to the benchmark. ${ }^{1}$ We contribute to the debate on asset managers' remuneration which is centered on two main issues: asset- vs. performance-based remuneration, asymmetric vs. fulcrum type fees.

To preserve tractability, we follow [Chen and Pennacchi(2009)] and we consider a class of remuneration schemes designed as a nonlinear function of the relative performance. The relative performance affects the remuneration either directly, through a performance fee which depends on the relative performance of the fund, or indirectly through the flow of funds in case of a remuneration provided by a management fee multiplying the assets under management (AUM). We provide conditions for unbounded excess risk taking when the relative performance tends to zero (gambling) and we characterize the investment strategy as the structure of the relative performance scheme varies. Our results suggest that the key point to prevent gambling is not the shape of a performance-based remuneration scheme (asymmetric vs. fulcrum fee) but rather the fact that manager's remuneration is sensitive to the relative performance in case of a very poor performance.

The possibility that a remuneration scheme may induce the asset manager to take risk in excess is a highly debated issue (see, for instance, [Gordon and Baptista(2010)] and references therein). The regulation of the mutual fund industry in US constrains the remuneration scheme of asset managers to be of fulcrum type (centered around an index with increases in fees for a performance above the index matched by decreases in fees for a performance below the index) under the assumption that an asymmetric convex remuneration scheme (call option on the performance upside) would induce the manager to take risk in excess. The claim was theoretically confirmed by two classical contributions: [Grinblatt and Titman(1989)], considering a risk neutral manager, and [Carpenter(2000)], in case of a risk averse manager. In the latter, it is shown that if the manager is remunerated through a call option then he/she tends to take unbounded risk with respect to the benchmark when the performance deteriorates significantly, whereas the tracking error is limited when the performance is above the strike.

A second generation of contributions casts doubts on this conclusion. Considering a remuneration scheme that is a nonlinear function of the relative performance, [Chen and Pennacchi(2009), Basak et al.(2007), Basak et al.(2008)] show that a remuneration scheme with downside risk (non bounded from below pay, liquidation risk, personal capital investment) may induce the asset manager to take less risk (smaller tracking error) when the relative performance tends to

\footnotetext{
1 We concentrate our attention on a partial equilibrium analysis (we take the remuneration contract as given), for a general equilibrium/principal-agent analysis of the remuneration of the asset manager we refer among the others to [Admati and Pfledeirer(1997), Das and Sundaram(2002), Lynch and Musto(1997), Ou-Yang(2003)].
} 
zero with risk shifting incentives in a finite range (when the performance of the fund is below but not too far away from the benchmark). ${ }^{2}$ The result has been confirmed by [Buraschi et al.(2014), Hodder and Jackwerth(2007), Dai and Sundaresan(2009)] assuming an absolute performance remuneration scheme. Also the empirical evidence is mixed: [Brown et al.(1996)] provide evidence that funds with poor performance are also characterized by higher variance of returns, [Busse(2001), Basak et al.(2007)] find no evidence. Finally, [Massa and Patgiri(2009)] show that convexity induces the manager to take more risk.

As in [Chen and Pennacchi(2009)], we consider remuneration schemes that include management/performance fees that are functions of the relative performance of the fund. We analyze various types of schemes: linear, convex, collar, convex-concave, concave-convex. We build upon a claim of [Basak et al.(2007)]: considering a particular class of remuneration schemes, they suggest that excess risk taking over a finite range occurs when the manager always incurs a penalty if the performance deteriorates and does not enjoy a fixed safety net independent of the fund value. We show two main results. First of all, considering a large class of remuneration schemes we qualify the above claim showing that the manager takes limited risk in excess when the relative performance of the fund tends to zero in case there is not a fixed fee and the remuneration is sensitive to the relative performance in a neighborhood of the origin. Second, differently from what is claimed by the regulation of mutual funds and by [Grinblatt and Titman(1989)], there is no significant difference between an asymmetric and a fulcrum type remuneration scheme with a fixed fee when the performance is poor, as a matter of fact the manager always tends to take risk in excess; however a difference is observed in case of a positive performance: in the first case the optimal strategy converges to the Merton solution, in the second case to the benchmark. Playing with the convexity and the concavity of the remuneration scheme, we show different shapes of the portfolio strategy, e.g. it can be either decreasing in the relative performance, increasing and then decreasing or decreasing, increasing and then decreasing. Note that these results hold true also considering an absolute performance fee (independent on the benchmark) as analyzed in [Grinblatt and Titman(1989), Carpenter(2000)].

We conclude our analysis with an empirical investigation on the mutual funds industry. Remember that managers of mutual funds are usually remunerated through a fixed fee and a management fee proportional to AUM, see [Golec(2003)]. Coherently with our analysis, and differently from [Basak et al.(2007)],

2 In the above papers, the remuneration is a function of the relative performance either through the flow of funds or directly through the remuneration scheme. In the first case, [Basak et al.(2007)] assume that the manager is remunerated through a coefficient (management fee) which applies to the AUM, the coefficient being a nonlinear function of the relative performance of the fund with respect to the benchmark. Using our notation -introduced in Section 2- the remuneration is of the form $F(T) P(X(T))$. See also [Nicolosi et al.(2017)]. [Chen and Pennacchi(2009)] directly consider that the AUM are a smooth concave increasing function of the relative performance of the fund, see also [Cuoco and Kaniel(2011), Koijen(2014)]. 
we show that there is a tendency to gambling with excess risk taking when the performance deteriorates significantly.

Our results show that unbounded (excess) risk taking does not occur when the relative performance tends to zero, if two conditions are met: the asset manager is not endowed with a fixed fee and the fee is sensitive to a very poor relative performance. This result agrees with $[\operatorname{Ross}(2004)]$, where the Author observes "to make agents more willing to take risks there should be more of a focus on offering downside protection than on offering them upside potential". Therefore, to prevent the manager to take risk in excess, a remuneration scheme based on a management fee proportional to the AUM or on a stake of the fund seems to perform better than a fixed fee and an incentive (symmetric or asymmetric) fee. Note that these results agree with empirical results obtained in [Aragon and Nanda(2014), Golec and Starks(2004)].

The paper is organized as follows. In Section 2 we introduce our setting. In Section 3 we analyze several different relative performance remuneration schemes, we derive the optimal portfolio solutions in closed form, and we compare the portfolio strategies. Finally, in Section 4 we present an empirical analysis on US equity mutual funds. All the proofs are postponed to the appendix.

\section{The model}

We consider a continuous time economy. There are two assets: the risk-free asset with a constant instantaneous interest rate $r$ and a risky asset. The risky asset price $S(t)$ evolves as

$$
d S(t)=S(t)(\mu d t+\sigma d Z(t)), \quad S(0)=S_{0}>0,
$$

where $\mu$ is the constant drift of the risky asset price, $\sigma$ is the constant volatility, and $Z(t)$ is a one-dimensional Brownian motion on a complete probability space $(\Omega, \mathcal{F}, P)$. We denote by $\mathcal{F}=\mathcal{F}_{t}$ the $P$-augmentation of the filtration generated by $Z(t)$.

The manager is remunerated evaluating his/her performance against a benchmark $Y(t)$ which is a portfolio with a fraction $\beta$ invested in the stock market and $(1-\beta)$ in the risk-free asset:

$$
d Y(t)=[(1-\beta) r+\beta \mu] Y(t) d t+\beta \sigma Y(t) d Z(t), \quad Y(0)=Y_{0}>0 .
$$

Given an adapted portfolio process $\theta(t)$, the performance of the fund is

$$
d F(t)=[(1-\theta(t)) r+\theta(t) \mu] F(t) d t+\theta(t) \sigma F(t) d Z(t), \quad F(0)=F_{0}>0
$$

we assume $F_{0}=Y_{0}$. To have a well-defined problem, we require

$$
\int_{0}^{T}(\theta(t) F(t))^{2} d t<+\infty \text { for any } T>0
$$


We consider the relative performance of the fund with respect to the benchmark

$$
X(t):=\frac{F(t)}{Y(t)},
$$

which evolves as

$$
d X(t)=X(t)\left[\delta(t)\left(\mu-r-\sigma^{2} \beta\right) d t+\delta(t) \sigma d Z(t)\right], \quad X(0)=1,
$$

with $\delta(t)=\theta(t)-\beta$ denoting the tracking error.

In the following we assume that the remuneration of the manager is defined over a finite horizon $T$ and that it depends on the terminal relative performance $X(T):=\frac{F(T)}{Y(T)}$. The manager defines the investment strategy $\theta(t)$ in order to maximize the expected utility of the remuneration. The utility function is of power type, i.e.

$$
u(x)=\frac{x^{a}}{a}, \quad a<1 .
$$

Note that $1-a$ represents the coefficient of relative risk aversion. The remuneration at time $T$ of the asset manager depends on the scheme which is a function of the relative performance at time $T: P(X(T))$.

\section{Relative Performance Remuneration Schemes}

We consider several types of remuneration schemes: linear, convex, collar, convex-concave, concave-convex. In detail we have the following schemes:

1. Linear: $P(X(T))=K+m X(T), K \geq 0, m>0$. The case $K=0$ corresponds to a remuneration which linearly depends only on the relative performance of the fund with respect to the benchmark and $m$ represents the sensitivity of the remuneration to the extra performance with respect to the benchmark. $K$ represents the fixed fee.

2. Convex (or incentive, or call option): $P(X(T))=K+(X(T)-1)^{+}, K \geq 0$. The manager receives a fixed fee $(K)$ and a variable component only in case the fund outperforms the benchmark at time $T(X(T) \geq 1)$.

3. Collar type (or capped call option): $P(X(T))=K+(X(T)-1)^{+}-(X(T)-$ $\mathcal{H})^{+}, K \geq 0, \mathcal{H}>1$. The manager receives a fixed fee $K$ and a variable component if the performance of the fund outperforms the benchmark $(X(T)>1)$ with a cap at $X(T)=\mathcal{H}>1$ (the maximum remuneration is $K+\mathcal{H}-1)$.

4. Convex-concave: $P(X(T))=K+m X(T)+p(X(T)-1)^{+}-c(X(T)-$ $\mathcal{H})^{+}, K, p, c \geq 0, m>0, m+p-c>0, \mathcal{H}>1$. The scheme is a combination of the linear and of the collar one. It is piecewise linear, the sensitivity of remuneration to the relative performance is low for a poor and for an outstanding performance compared to an intermediate performance $(1 \leq$ $X(T) \leq \mathcal{H})$. 

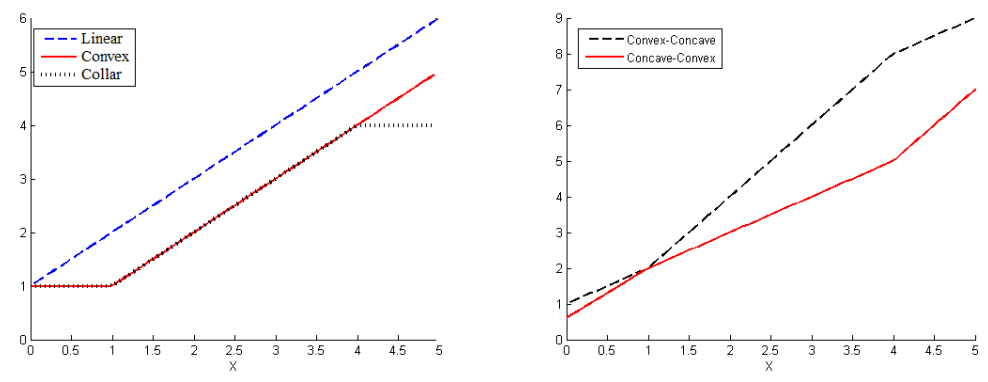

Fig. 1 Remuneration schemes

5. Concave-convex: $P(X(T))=K+m X(T)+p\left(X(T)-H_{1}\right)^{+}-c\left(H_{2}-\right.$ $X(T))^{+}, K, p, c, m \geq 0, \min \{m+p, m+c\}>0, H_{1}>H_{2}>0, K-c H_{2} \geq$ 0 . The scheme is specular to the convex-concave. It is piecewise linear, the sensitivity of remuneration to the relative performance is high for a poor and for an outstanding performance compared to an intermediate performance.

The payoffs of the remuneration schemes are depicted in Figure 1. Note that the last two schemes are similar to those analyzed in [Basak et al.(2007), Basak et al.(2008)] but are not included in their framework. The analysis developed below holds true also in case the above remuneration schemes are defined on the absolute performance $F(T)$.

\subsection{Linear scheme}

The manager aims at solving the following problem:

$$
\max _{\theta} E[u(K+m X(T))]
$$

for any admissible strategy $\theta(t)$, where the process $(X(t))_{t \geq 0}$ is defined in Equation (2). The above problem is related to the following value function

$$
V(t, x)=\max _{\delta} E[u(K+m X(T)) \mid X(t)=x],
$$

where we maximize over the tracking error $\delta=\theta-\beta$. This problem can be solved via the martingale technique, obtaining a closed form solution.

Theorem 1 Assuming $K>0$, the optimal tracking error is

$$
\delta^{*}(t)=\frac{\vartheta}{\sigma(1-a)}\left[1+\frac{K}{m} \frac{N\left(d_{1}(t)\right)}{X^{*}(t)}\right],
$$

where $X^{*}(t)$ denotes the optimal relative performance which evolves as

$$
X^{*}(t)=\frac{K}{m}\left(\frac{N^{\prime}\left(d_{1}(t)\right)}{N^{\prime}\left(d_{2}(t)\right)} N\left(d_{2}(t)\right)-N\left(d_{1}(t)\right)\right),
$$



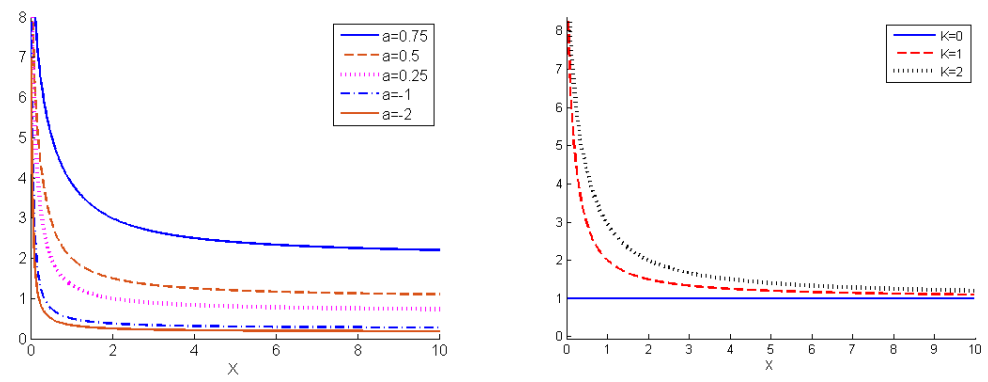

Fig. 2 Optimal tracking error $\delta^{*}$ for the linear scheme. Parameters: $T=2 ; t=1 ; \mu-r=0.03$; $\sigma=0.2 ; a=0.5 ; \beta=0.25 ; m=1 ; K=1$.

with

$$
\vartheta=\frac{\mu-r-\sigma^{2} \beta}{\sigma}
$$

and $N(\cdot)$ being the standard cumulative normal distribution (all the other coefficients are defined in the appendix).

Figure 2 (right) shows the optimal strategy varying $K$.

If $K=0$, we go back to the classical Merton problem with terminal utility, and we obtain the constant optimal tracking error (Merton solution):

$$
\delta^{*}=\frac{\mu-r-\beta \sigma^{2}}{(1-a) \sigma^{2}},
$$

i.e. Equation (3) with $K=0$. We observe that in case $\mu-r-\beta \sigma^{2}>0(<0)$ the optimal investment strategy $\theta^{*}=\beta+\delta^{*}$ is overinvested (underinvested) in the risky asset with respect to the benchmark. In what follows, we always assume parameter values such that $\mu-r-\beta \sigma^{2}>0$.

A fixed fee $K>0$ induces the manager to take excess (unbounded) risk when the relative performance of the fund deteriorates: the investment strategy is decreasing in $X$, it becomes unbounded as $X \rightarrow 0$, with the same sign as $\mu-r-\beta \sigma^{2}$, and the tracking error converges to the one obtained for $K=0$ $\left(\delta^{*}=\vartheta /(\sigma(1-a))\right)$ as $X \rightarrow \infty$. As $K$ increases, the tracking error increases, i.e. a higher floor induces the manager to take more risk. Figure 2 (left) shows the optimal strategies varying $a$ : the tracking error $\delta^{*}$ explodes as the manager's risk tolerance increases $(a \rightarrow 1)$ and it converges to zero in case risk aversion increases $(a \rightarrow-\infty)$, the result agrees with the analysis in $[\operatorname{Koijen}(2014)]$.

\subsection{Convex scheme}

As in [Grinblatt and Titman(1989)] and [Carpenter(2000)], we assume that the manager receives a fixed fee $(K)$ and a variable component only in case 

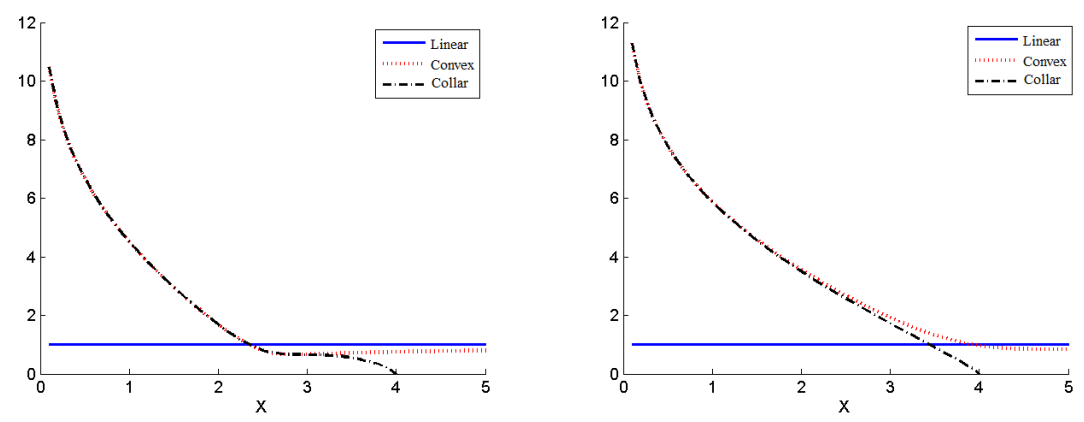

Fig. 3 Optimal tracking error $\delta^{*}$ for the linear, convex (option type) and capped (collar type) scheme. Parameters: $T=2 ; t=1 ; \mu-r=0.03 ; \sigma=0.2 ; a=0.5 ; \beta=0.25$. For the linear scheme we set $m=1, K=0$. For the other schemes we set $K=0$ (left), $K=0.2$ (right), $\mathcal{H}=4$.

the fund performance outperforms the benchmark at time $T(X(T) \geq 1)$. We model this component through a call option on $X(T)$ with strike price 1 .

The asset manager aims at solving the following problem

$$
\max _{\theta} E\left[u\left((X(T)-1)^{+}+K\right)\right]
$$

subject to (2). Notice that $K \geq 0$, however, the additional condition $a \in$ $(0,1)$ is required if $K=0$. The problem is similar to the one analyzed in [Carpenter(2000)] and the following result holds true.

Theorem 2 The optimal tracking error is

$$
\delta^{*}(t)=\frac{\vartheta}{\sigma X^{*}(t)}\left[\frac{X^{*}(t)}{1-a}+\frac{\widehat{x} N^{\prime}\left(d_{1}(t)\right)}{|\vartheta| \sqrt{T-t}}-(1-K) \frac{N\left(d_{1}(t)\right)}{1-a}\right],
$$

where

$$
X^{*}(t)=(1-K) N\left(d_{1}(t)\right)+(\widehat{x}-1+K) \frac{N^{\prime}\left(d_{1}(t)\right)}{N^{\prime}\left(d_{2}(t)\right)} N\left(d_{2}(t)\right)
$$

(all the other coefficients are defined in the appendix).

The optimal tracking error is depicted in Figures 3 and 4 (dotted curve) and is similar to the one obtained in [Carpenter(2000)]. Notice that as $X \rightarrow 0$, $\left|\delta^{*}\right|$ converges to infinity with a sign in agreement with the one of $\mu-r-\beta \sigma^{2}$. On the other hand, for large values of $X$ the optimal strategy approaches the constant strategy obtained in case of a linear scheme with $K=0$. The rationale of this shape is that for $X$ large enough the remuneration is well approximated by the linear scheme $(m=1, K=0)$. Confirming what we have observed for the linear case, in Figure 3 we show that the higher is the fixed fee $K$ the higher is the risk exposure. Risk exposure is also decreasing in risk aversion, see Figure 4. 



Fig. 4 Optimal tracking error $\delta^{*}$ for the linear, convex (option type) and capped (collar type) scheme. Parameters: $T=2 ; t=1 ; \mu-r=0.03 ; \sigma=0.2 ; \beta=0.25 ; a=0.5$ (left), $a=-0.5$ (right). For the linear scheme we set $m=1, K=0$. For the other schemes we set $K=0.3, \mathcal{H}=4$.

\subsection{Collar type scheme}

We follow [Grinblatt and Titman(1989)] considering a collar type remuneration scheme as the simplest fulcrum fee. The manager aims at solving the following problem

$$
\max _{\theta} E\left[u\left((X(T)-1)^{+}-(X(T)-\mathcal{H})^{+}+K\right)\right]
$$

subject to (2), assuming $\mathcal{H}>1$. Without loss of generality, we assume a hurdle at 1 . Again, the additional condition $a \in(0,1)$ is required if $K=0$. The following result holds true.

Theorem 3 Assume $\mathcal{H}>\widehat{x}>1$, then, for any $t \in(0, T]$ such that

$$
\max _{s \in[0, t)} X^{*}(s)<\mathcal{H}
$$

the optimal tracking error is

$\delta^{*}(t)=\frac{\vartheta}{\sigma X^{*}(t)}\left[\frac{X^{*}(t)}{1-a}+\frac{\widehat{x} N^{\prime}\left(d_{3}(t)\right)}{|\vartheta| \sqrt{T-t}}-(1-K) \frac{N\left(d_{3}(t)\right)}{1-a}-(\mathcal{H}-1+K) \frac{N\left(d_{1}(t)\right)}{1-a}\right]$,

where

$$
\begin{aligned}
X^{*}(t) & =(1-K) N\left(d_{3}(t)\right)+(\widehat{x}-1+K) \frac{N^{\prime}\left(d_{3}(t)\right)}{N^{\prime}\left(d_{4}(t)\right)} N\left(d_{4}(t)\right) \\
& +(\mathcal{H}-1+K)\left[N\left(d_{1}(t)\right)-\frac{N^{\prime}\left(d_{1}(t)\right)}{N^{\prime}\left(d_{2}(t)\right)} N\left(d_{2}(t)\right)\right]
\end{aligned}
$$

(all coefficients are defined in the appendix). 
Condition (8) is due to the fact that if there exists a time instant $t \in[0, T]$ such that $X^{*}(t)=\mathcal{H}$, then the optimal strategy is trivial: for any $s \in[t, T]$, $X^{*}(s)=\mathcal{H}, \delta^{*}(s)=0$.

In Figures 3 and 4 we plot the optimal tracking error $\delta^{*}$ considering the three remuneration schemes analyzed above. Note that the optimal tracking errors for the convex and for the collar type remuneration scheme are similar in case of a poor performance (flat fee) and in the interval with a remuneration linear in the relative performance $\left(1 \leq X^{*}(t) \leq \mathcal{H}\right)$ : the shape is decreasing with unlimited exposure in case the relative performance approaches 0 . Instead, they look different when the cap is reached: the optimal strategy for a collar scheme exhibits a zero tracking error, the optimal strategy for a call option converges to the one obtained in case of a linear scheme. The rationale is very simple: if the manager reaches the cap to its remuneration, then he/she sticks to the benchmark. As for a convex scheme, in case of a collar type scheme risk exposure goes up with the safety net $K$ and as the risk aversion decreases.

Although the relative performance remuneration scheme is similar to the one considered in [Basak et al.(2007)], the optimal strategy looks quite different. In [Basak et al.(2007)] the expected utility is a function of the AUM that are obtained by multiplying (according to our notation) $P(X(T))$ by $F(T)$ (asset-based fee). In that framework, excess risk taking is observed over a finite range, there is no unlimited risk exposure in case of a very poor performance, and when the relative performance reaches the cap the optimal strategy tends to the Merton solution. The fact that the nonlinear relative performance function multiplies the funds (linear term) drives the results: in [Basak et al.(2007)] the remuneration is always sensitive to the fund performance, whereas in our setting it is flat in case of a poor and of an outstanding performance.

We can conclude that when a fulcrum type remuneration scheme of the relative performance is considered there is an incentive to gambling in a neighborhood of the origin. This result is at odds with the analysis of [Grinblatt and Titman(1989)] and with the insights of the regulation of the mutual fund industry.

\subsection{Convex-concave scheme}

We assume that the manager aims at solving the following problem

$$
\max _{\theta} E\left[u\left((X(T)-1)^{+}-(X(T)-\mathcal{H})^{+}+m X(T)+K\right)\right],
$$

subject to (2). Without loss of generality we have set $p=c=1$. Note that remuneration is piecewise linear in the relative performance and the sensitivity of the remuneration is $m$ for $X(T)<1$ and for $X(T)>\mathcal{H}$ and is $m+1$ for $1<X(T)<\mathcal{H},{ }^{3}$ i.e. sensitivity is higher just above the benchmark. Note

\footnotetext{
3 In the general case, the sensitivity of the remuneration is $m$ for $X(T)<1, m+p-c$ for $X(T)>H$ and is $m+p$ for $1<X(T)<H$
} 

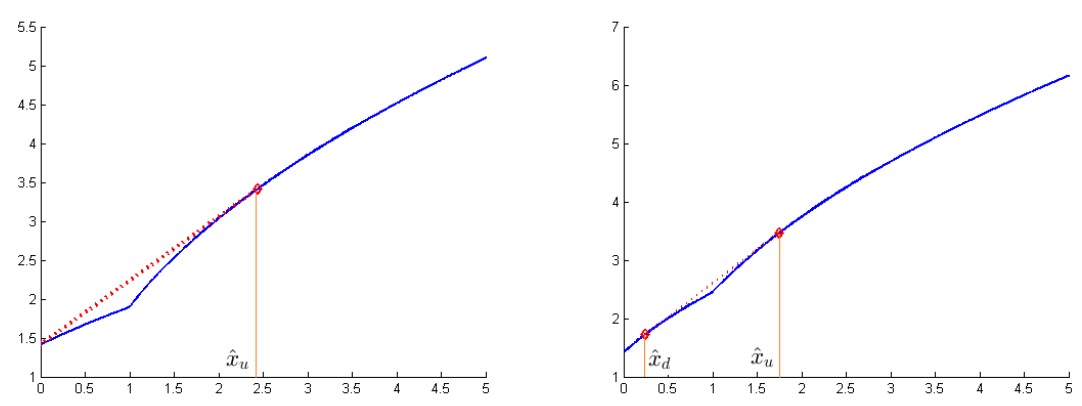

Fig. 5 Concavification. $\mathcal{H}=5, a=K=0.5, m=0.4$ (left), $m=1$ (right)

that the shape of the remuneration scheme is convex and then concave in the relative performance.

In order to obtain the unique concavification, two assumptions should be considered. Notice that they can not be satisfied together.

Assumption 1 There exists $\widehat{x}_{u} \in(1, \mathcal{H}]$ such that:

$\rightarrow U^{\prime}\left(\widehat{x}_{u}\right)=\frac{U\left(\widehat{x}_{u}\right)-U(0)}{\widehat{x}_{u}}$

$\rightarrow U(x) \leq \frac{U\left(\widehat{x}_{u}\right)-U(0)}{\widehat{x}_{u}} x+U(0)$ for any $x \in\left[0, \widehat{x}_{u}\right]$;

being $K>0$ if $a$ is negative, i.e. $U(0)$ is well-defined.

Assumption 2 There exist $0<\widehat{x}_{d}<1<\widehat{x}_{u} \leq \mathcal{H}$ such that:

$\rightarrow U^{\prime}\left(\widehat{x}_{u}\right)=U^{\prime}\left(\widehat{x}_{d}\right)=\frac{U\left(\widehat{x}_{u}\right)-U\left(\widehat{x}_{d}\right)}{\widehat{x}_{u}-\widehat{x}_{d}} ;$

$\rightarrow U(x) \leq \frac{U\left(\widehat{x}_{u}\right)-U\left(\widehat{x}_{d}\right)}{\widehat{x}_{u}-\widehat{x}_{d}}\left(x-\widehat{x}_{d}\right)+U\left(\widehat{x}_{d}\right)$ for any $x \in\left[\widehat{x}_{d}, \widehat{x}_{u}\right]$.

The two assumptions are related to the two cases depicted in Figure 5.

The following results hold true.

Theorem 4 Let us assume that Assumption 1 holds true. Then the optimal tracking error is

$$
\begin{aligned}
\delta^{*}(t) & =\frac{\vartheta}{\sigma X^{*}(t)}\left(\frac{X^{*}(t)}{1-a}+\widehat{x}_{u} \frac{N^{\prime}\left(d_{5}(t)\right)}{|\vartheta| \sqrt{T-t}}-\frac{1-K}{1+m} \frac{N\left(d_{5}(t)\right)-N\left(d_{3}(t)\right)}{1-a}\right. \\
& \left.-\mathcal{H} \frac{N\left(d_{3}(t)\right)-N\left(d_{1}(t)\right)}{1-a}-\frac{1-K-\mathcal{H}}{m} \frac{N\left(d_{1}(t)\right)}{1-a}\right),
\end{aligned}
$$

with

$$
\begin{aligned}
X^{*}(t) & =\frac{\widehat{x}_{u}(1+m)+K-1}{1+m} \frac{N^{\prime}\left(d_{5}(t)\right)}{N^{\prime}\left(d_{6}(t)\right)} N\left(d_{6}(t)\right)-\frac{\mathcal{H}(1+m)+K-1}{1+m} \frac{N^{\prime}\left(d_{3}(t)\right)}{N^{\prime}\left(d_{4}(t)\right)} N\left(d_{4}(t)\right) \\
& +\frac{1-K}{1+m}\left(N\left(d_{5}(t)\right)-N\left(d_{3}(t)\right)\right)+\mathcal{H}\left(N\left(d_{3}(t)\right)-N\left(d_{1}(t)\right)\right) \\
& +\frac{\mathcal{H}(1+m)+K-1}{m} \frac{N^{\prime}\left(d_{1}(t)\right)}{N^{\prime}\left(d_{2}(t)\right)} N\left(d_{2}(t)\right)+\frac{1-K-\mathcal{H}}{m} N\left(d_{1}(t)\right)
\end{aligned}
$$

(all coefficients are defined in the appendix). 
Theorem 5 Let us assume that Assumption 2 holds true and $K>0$. Then the optimal tracking error is

$$
\begin{aligned}
\delta^{*}(t) & =\frac{\vartheta}{\sigma X^{*}(t)}\left(\frac{X^{*}(t)}{1-a}+\left(\widehat{x}_{u}-\widehat{x}_{d}\right) \frac{N^{\prime}\left(d_{5}(t)\right)}{|\vartheta| \sqrt{T-t}}+\frac{K}{m} \frac{\left(N\left(d_{7}(t)\right)-N\left(d_{5}(t)\right)\right)}{1-a}\right. \\
& \left.-\frac{1-K}{1+m} \frac{N\left(d_{5}(t)\right)-N\left(d_{3}(t)\right)}{1-a}-\mathcal{H} \frac{N\left(d_{3}(t)\right)-N\left(d_{1}(t)\right)}{1-a}-\frac{1-K-\mathcal{H}}{m} \frac{N\left(d_{1}(t)\right)}{1-a}\right),
\end{aligned}
$$

with

$$
\begin{aligned}
X^{*}(t) & =\frac{K}{m} \frac{N^{\prime}\left(d_{7}(t)\right)}{N^{\prime}\left(d_{8}(t)\right)} N\left(d_{8}(t)\right)-\frac{m \widehat{x}_{d}+K}{m} \frac{N^{\prime}\left(d_{5}(t)\right)}{N^{\prime}\left(d_{6}(t)\right)} N\left(d_{6}(t)\right)-\frac{K}{m}\left(N\left(d_{7}(t)\right)-N\left(d_{5}(t)\right)\right) \\
& +\frac{\widehat{x}_{u}(1+m)+K-1}{1+m} \frac{N^{\prime}\left(d_{5}(t)\right)}{N^{\prime}\left(d_{6}(t)\right)} N\left(d_{6}(t)\right)-\frac{\mathcal{H}(1+m)+K-1}{1+m} \frac{N^{\prime}\left(d_{3}(t)\right)}{N^{\prime}\left(d_{4}(t)\right)} N\left(d_{4}(t)\right) \\
& +\frac{1-K}{1+m}\left(N\left(d_{5}(t)\right)-N\left(d_{3}(t)\right)\right)+\mathcal{H}\left(N\left(d_{3}(t)\right)-N\left(d_{1}(t)\right)\right) \\
& +\frac{\mathcal{H}(1+m)+K-1}{m} \frac{N^{\prime}\left(d_{1}(t)\right)}{N^{\prime}\left(d_{2}(t)\right)} N\left(d_{2}(t)\right)+\frac{1-K-\mathcal{H}}{m} N\left(d_{1}(t)\right)
\end{aligned}
$$

(all coefficients are defined in the appendix).

Remark 1 If $K=0$, Theorem 5 holds true with Equation (11) replaced by

$$
\begin{aligned}
X^{*}(t) & =\frac{1}{m}\left(\frac{\lambda \xi(t)}{m}\right)^{1 /(a-1)} e^{\frac{a \vartheta^{2}}{2(1-a)^{2}}(T-t)}-\widehat{x}_{d} \frac{N^{\prime}\left(d_{5}(t)\right)}{N^{\prime}\left(d_{6}(t)\right)} N\left(d_{6}(t)\right) \\
& +\frac{\widehat{x}_{u}(1+m)-1}{1+m} \frac{N^{\prime}\left(d_{5}(t)\right)}{N^{\prime}\left(d_{6}(t)\right)} N\left(d_{6}(t)\right)-\frac{\mathcal{H}(1+m)-1}{1+m} \frac{N^{\prime}\left(d_{3}(t)\right)}{N^{\prime}\left(d_{4}(t)\right)} N\left(d_{4}(t)\right) \\
& +\frac{1}{1+m}\left(N\left(d_{5}(t)\right)-N\left(d_{3}(t)\right)\right)+\mathcal{H}\left(N\left(d_{3}(t)\right)-N\left(d_{1}(t)\right)\right) \\
& +\frac{\mathcal{H}(1+m)-1}{m} \frac{N^{\prime}\left(d_{1}(t)\right)}{N^{\prime}\left(d_{2}(t)\right)} N\left(d_{2}(t)\right)+\frac{1-\mathcal{H}}{m} N\left(d_{1}(t)\right) .
\end{aligned}
$$

Notice that the above equation and Equation (11) are related, since

$$
\lim _{K \rightarrow 0} K \frac{N^{\prime}\left(d_{7}(t)\right)}{N^{\prime}\left(d_{8}(t)\right)} N\left(d_{8}(t)\right)=\left(\frac{\lambda \xi(t)}{m}\right)^{1 /(a-1)} e^{\frac{a \vartheta^{2}}{2(1-a)^{2}}(T-t)} .
$$

In Figure 6 we plot the optimal tracking error for the collar and the convexconcave scheme considering two different values of $K$. These figures highlight the role of a linear component $(m \neq 0)$, which is absent in the convex and in the collar type scheme, and of a fixed floor $(K \geq 0)$. We recall that the collar type fee corresponds to the convex-concave case with $m=0$ and $K \geq 0$. We concentrate our attention on a performance smaller than $\mathcal{H}$. If $K>0$ and $m \neq 0$, for all set of parameters (including the cases $p, c \neq 1$ ), then the optimal tracking error is decreasing in the performance and tends to become unbounded as $X \rightarrow 0$, yielding excessive risk taking. Instead, if $K=0$ and $m \neq 0$, then the optimal tracking error is hump shaped and converges to the 

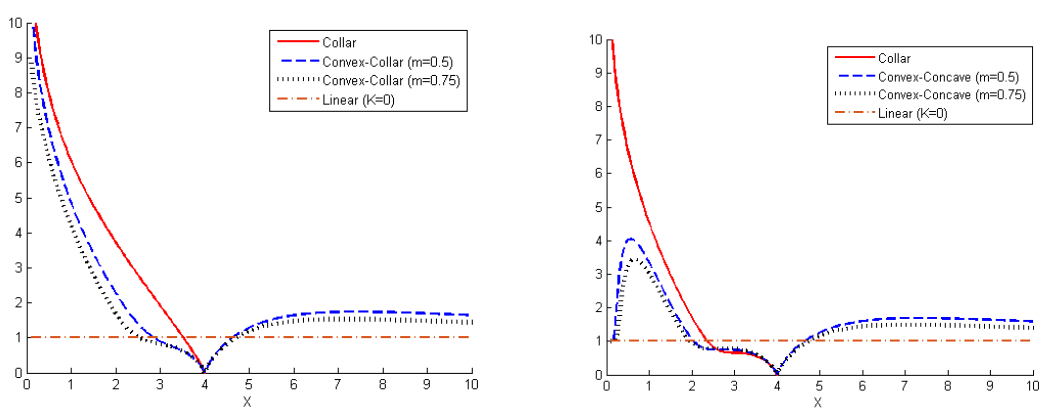

Fig. 6 Optimal tracking error $\delta^{*}$ for the collar and convex-concave remuneration scheme. Parameters: $T=2 ; t=1 ; \mu-r=0.03 ; \sigma=0.2 ; \beta=0.25 ; \mathcal{H}=4 ; a=0.5 ; K=0.3$ (left) $K=0$ (right).

Merton solution (5) as $X \rightarrow 0$. Note that the analysis of the collar scheme shows that the first shape of the optimal strategy is also obtained with $K \geq 0$ and $m=0$, instead the shape of the optimal strategy obtained for $K=0$ and $m \neq 0$ is the one of [Basak et al.(2007)]. These results confirm that the presence of a strictly positive safety net $K>0$ induces the manager to take unlimited risk when the relative performance tends to zero.

\subsection{Concave-convex remuneration scheme}

We consider the payoff analyzed in [Buraschi et al.(2014)] for an absolute performance fee, moved to our framework: the manager aims at solving the following problem

$$
\max _{\theta} E\left[u\left(p\left(X(T)-H_{1}\right)^{+}+m X(T)-c\left(H_{2}-X(T)\right)^{+}+K\right)\right],
$$

with $H_{1}>H_{2}>0$ and $K-c H_{2} \geq 0$. Again, in this case, two assumptions should be considered. ${ }^{4}$

Assumption 3 There exists $\widehat{x}_{u}>H_{1}$ such that:

$\rightarrow U^{\prime}\left(\widehat{x}_{u}\right)=\frac{U\left(\widehat{x}_{u}\right)-U(0)}{\widehat{x}_{u}} ;$
$\rightarrow U(x) \leq \frac{U\left(\widehat{x}_{u}\right)-U(0)}{\widehat{x}_{u}} x+U(0)$ for any $x \in\left[0, \widehat{x}_{u}\right]$,

being $\mathrm{K}-\mathrm{cH}_{2}>0$ if $a$ is negative, i.e. $U(0)$ is well-defined.

Assumption 4 There exist $0<\widehat{x}_{d}<H_{2}<H_{1}<\widehat{x}_{u}$ such that:

4 As an example, Assumption 3 holds true if $a=-0.5 ; m=0.02 ; K=0.5 ; c=0.02 ; H_{1}=$ $5 ; H_{2}=3 ; p=0.2$. If $K=0.2$ Assumption 4 holds true (other parameters as above). Moreover, if $K=0.08$ none of the two assumptions hold true: in this case a concavification is still possible in the sense of Assumption 4, with $\widehat{x}_{d}=H_{2}$, and with a concavified utility function with a first order derivative not well defined in $H_{2}$ (see [Buraschi et al.(2014)] for further details). 
$\rightarrow U^{\prime}\left(\widehat{x}_{u}\right)=U^{\prime}\left(\widehat{x}_{d}\right)=\frac{U\left(\widehat{x}_{u}\right)-U\left(\widehat{x}_{d}\right)}{\widehat{x}_{u}-\widehat{x}_{d}} ;$

$\rightarrow U(x) \leq \frac{U\left(\widehat{x}_{u}\right)-U\left(\widehat{x}_{d}\right)}{\widehat{x}_{u}-\widehat{x}_{d}}\left(x-\widehat{x}_{d}\right)+U\left(\widehat{x}_{d}\right)$ for any $x \in\left[\widehat{x}_{d}, \widehat{x}_{u}\right]$.

The following results hold true.

Theorem 6 Let us assume that Assumption 3 holds true. Then the optimal tracking error is

$$
\delta^{*}(t)=\frac{\vartheta}{\sigma X^{*}(t)}\left(\frac{X^{*}(t)}{1-a}+\frac{\widehat{x}_{u} N^{\prime}\left(d_{1}(t)\right)}{|\vartheta| \sqrt{T-t}}-\frac{p H_{1}-K}{m+p} \frac{N\left(d_{1}(t)\right)}{1-a}\right),
$$

with

$$
X^{*}(t)=\frac{p H_{1}-K}{m+p} N\left(d_{1}(t)\right)+\frac{\widehat{x}_{u}(m+p)+K-p H_{1}}{m+p} \frac{N^{\prime}\left(d_{1}(t)\right)}{N^{\prime}\left(d_{2}(t)\right)} N\left(d_{2}(t)\right)
$$

(all coefficients are defined in the appendix).

Theorem 7 Let us assume that Assumption 4 holds true and $\mathrm{K}-\mathrm{cH}_{2}>0$. Then the optimal tracking error is

$$
\begin{aligned}
\delta^{*}(t) & =\frac{\vartheta}{\sigma X^{*}(t)}\left(\left(\widehat{x}_{u}-\widehat{x}_{d}\right) \frac{N^{\prime}\left(d_{1}(t)\right)}{|\vartheta| \sqrt{T-t}}\right. \\
& \left.+\frac{X^{*}(t)}{1-a}-\frac{p H_{1}-K}{m+p} \frac{N\left(d_{1}(t)\right)}{1-a}-\frac{c H_{2}-K}{m+c} \frac{N\left(d_{3}(t)\right)-N\left(d_{1}(t)\right)}{1-a}\right),
\end{aligned}
$$

with

$$
\begin{aligned}
X^{*}(t) & =\frac{\widehat{x}_{u}(m+p)+K-p H_{1}}{m+p} \frac{N^{\prime}\left(d_{1}(t)\right)}{N^{\prime}\left(d_{2}(t)\right)} N\left(d_{2}(t)\right)+\frac{p H_{1}-K}{m+p} N\left(d_{1}(t)\right) \\
& +\frac{1}{m+c}\left(\left(K-c H_{2}\right) \frac{N^{\prime}\left(d_{3}(t)\right)}{N^{\prime}\left(d_{4}(t)\right)} N\left(d_{4}(t)\right)-\left((m+c) \widehat{x}_{d}+K-c H_{2}\right) \frac{N^{\prime}\left(d_{1}(t)\right)}{N^{\prime}\left(d_{2}(t)\right)} N\left(d_{2}(t)\right)\right) \\
& +\frac{c H_{2}-K}{m+c}\left(N\left(d_{3}(t)\right)-N\left(d_{1}(t)\right)\right)
\end{aligned}
$$

(all coefficients are defined in the appendix).

Remark 2 If $\mathrm{K}=\mathrm{cH}_{2}$, Theorem 7 holds true with Equation (13) replaced by

$$
\begin{aligned}
X^{*}(t) & =\frac{\widehat{x}_{u}(m+p)-p H_{1}}{m+p} \frac{N^{\prime}\left(d_{1}(t)\right)}{N^{\prime}\left(d_{2}(t)\right)} N\left(d_{2}(t)\right)+\frac{p H_{1}}{m+p} N\left(d_{1}(t)\right) \\
& +\frac{1}{m+c}\left(\left(\frac{\lambda \xi(t)}{m+c}\right)^{\frac{1}{a-1}} e^{\frac{a \vartheta^{2}}{2(1-a)^{2}}(T-t)}-\left((m+c) \widehat{x}_{d}-c H_{2}\right) \frac{N^{\prime}\left(d_{1}(t)\right)}{N^{\prime}\left(d_{2}(t)\right)} N\left(d_{2}(t)\right)\right) \\
& +\frac{c H_{2}}{m+c}\left(N\left(d_{3}(t)\right)-N\left(d_{1}(t)\right)\right) .
\end{aligned}
$$





Fig. 7 Optimal tracking error $\delta^{*}$ for the concave-convex remuneration scheme, varying $m$ and $H_{1}$. Parameters: $T=2 ; t=1 ; \mu-r=0.03 ; \sigma=0.2 ; \beta=0.25 ; a=0.5 ; c=0.02 ; H_{1}=$ $4 ; H_{2}=1 ; p=0.75 ; m=0.3$ and $K=0.2$.

We start by assuming $\mathrm{K}-\mathrm{cH}_{2}>0$. Note that this corresponds to the hypothesis of a strictly positive floor. In Figure 7 we plot the optimal investment strategy varying $m, H_{1}, c$ and $K$. We would like to stress that Assumption 3 holds true only in the first figure (up-left), whereas Assumption 4 is satisfied in the other three cases. We observe that, as for the other remuneration schemes, the optimal tracking error converges to $\infty$ when the relative performance converges to zero, whereas the strategy converges to the optimal strategy obtained in the linear case for a relative performance high enough. In the transition, the shape can be either decreasing or decreasing-increasingdecreasing with a relative minimum and a relative maximum (hump shaped).

The non monotonic shape is due to the fact that the scheme is concave for a low relative performance. If this feature plays a relevant role, then the asset manager may take a limited risk exposure in case of a poor (but not extremely poor) performance. This interpretation is confirmed by the comparative statics analysis. If $m$ or $H_{1}$ are small enough, or $K$ is large enough, then the optimal strategy is decreasing as in the case of a convex scheme, otherwise the optimal strategy exhibits a local minimum and a local maximum. The interpretation is as follows. When the second kink at $H_{1}$ inducing convexity is high enough, we may observe a limited risk exposure for a poor performance (the concave part of the scheme plays a role). As far as the fixed fee $K$ is concerned, we confirm that a large fixed fee induces the manager to take a significant risk exposure and this effect is likely to induce a monotonic decreasing shape. 

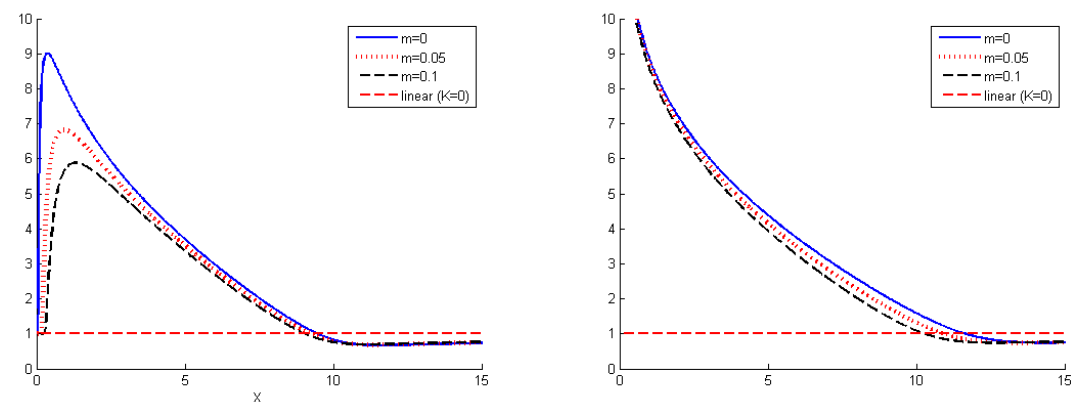

Fig. 8 Optimal tracking error $\delta^{*}$ for the concave-convex remuneration scheme. Parameters: $T=2 ; t=1 ; \mu-r=0.03 ; \sigma=0.2 ; \beta=0.25 ; a=0.5 ; c=0.02 ; H_{1}=4 ; H_{2}=1 ; p=0.75$; and $\mathrm{K}=\mathrm{cH}_{2}$ (left), $\mathrm{K}=\mathrm{cH}_{2}+0.1$ (right).

The shape of the optimal strategy changes significantly in case $\mathrm{K}-\mathrm{cH}_{2}=0$ (zero floor). As shown in Figure 8, if the floor is null (left panel) then the optimal strategy is characterized by excess risk taking over a finite interval (hump shaped strategy); instead if there is a strictly positive floor to the remuneration (right panel) then the asset manager takes an unlimited risk exposure as the relative performance tends to zero.

\section{Empirical Analysis}

The above theoretical analysis provides us with a research question that is worthwhile to investigate: Do relative performance remuneration schemes provide an incentive to take unbounded risk (gambling) when the relative performance deteriorates $\left(H p^{0}\right)$, as suggested by [Carpenter(2000)], or to take risk in excess only over a finite range as suggested by [Basak et al.(2007)] $\left(H p^{1}\right)$ ?

We address this question analyzing the performance of mutual funds replicating the analysis developed in [Basak et al.(2007)]. We focus our analysis on US equity mutual funds and in particular we select the funds active in the period 1995- 2017 more exposed to equity-based active management, i.e. those classified as Capital Appreciation, Growth and Income or Growth Funds within the Lipper Funds Classification.

Starting from this list of funds, we construct a database of daily observed funds' Net Asset Values as available from the data provider Thomson Reuters Datastream. Funds with less than 250 observations are excluded from the sample. As standard practice in literature, we use the S\&P500 index as benchmark for all funds and the interest rate on 3 months T-bills as proxy of risk-free rate.

For each year we restrict our analysis to those funds with beta higher than one. In [Christoffersen and Simutin(2016)], authors show that funds more susceptible to benchmarking pressures increase their demand for high beta stocks reducing their exposure to low-beta ones. This observation suggests to restrict our attention to funds with a beta higher than one. For each year in 
the sample, then, we discriminate the funds accordingly to the beta estimated using daily observations of risk-free rate, funds and benchmark returns. For the sake of completeness, along with the results for the subset of funds with beta higher than one, we also present the evidence obtained for the full sample of funds.

Note that in the mutual fund industry the remuneration scheme usually consists in a fixed fee and in a management fee proportional to AUM, see [Golec(2003)]. According to our analysis we should find evidence in favor of $H p^{0}$. We want to analyze how different levels of relative performance affect the manager's risk-shifting incentives.

We first focus on underperforming funds (Table 1). At a monthly level we split the range of underperformance with respect to the benchmark into mutually exclusive intervals delimited by the reference levels $B=\{0 \%, 5 \%, 10 \%, 15 \%, 20 \%\}$.

That is, we associate, for example, a $6.5 \%$ monthly underperformance to the $5 \%$-interval. The last interval, i.e. the one bounded from above by $-20 \%$, contains all funds with monthly underperformance equal to or smaller than $-20 \%$. For each interval we create a corresponding dummy variable $U N D E R(b)_{i, m}$ which is equal to one if the fund's relative performance in the preceding month is in the $b$-interval and zero otherwise.

Under $H p^{0}$ we expect that the farther a fund falls behind the benchmark, the higher is the incentive for the manager to take risk in excess, i.e. the larger is the stake in the benchmark and therefore the higher is the sensitivity of the fund with respect to the benchmark. As shown in Section 3, this effect is obtained for all the remuneration schemes, except in case there is no fixed fee and the remuneration is sensitive to the performance in a neighborhood of the origin. In the latter case, risk exposure is likely to be hump shaped: limited exposure when the relative performance is significantly poor, significant exposure for intermediate poor relative performance, again limited exposure when the performance falls behind the benchmark but not too much.

To test the two hypotheses, we regress the daily fund's excess returns $R_{i, t}^{F}-r_{t}$ on the S\&P500 index's daily excess returns $R_{t}^{B}-r_{t}$ interacted with the $U N D E R$ indicators defined above. Performing this regression we implicitly assume that the benchmark of the funds is related to the S\&P500 index. Here $r_{t}$ denotes the risk-free rate observed on day $t$. We also include year and month fixed effects to address the seasonality in betas, see for example [Lewellen and Nagel(2006)]. Regression results are shown in Table 1. In both cases (i.e. full sample and funds with beta higher than one), estimated coefficients are all positive and increasing in the magnitude of underperformance: funds falling far below the benchmark at the end of a month tend to take excess risk in the subsequent month by increasing their investment in the risky assets and this leads to an increase in its sensitivity with respect to the benchmark compared to funds with better relative performance. This evidence is in favour of the unbounded risk taking incentive hypothesis ${ }^{5} \mathrm{Hp}^{0}$. Indeed, gambling in-

5 For the sake of completeness, we point out that these results are not directly comparable to those obtained in [Basak et al.(2007)] where the findings support the hypothesis $H p^{1}$. 


\begin{tabular}{lcc}
\hline & \multicolumn{2}{c}{ Dependent Variable: $R_{i, t}^{F}-r_{t}$} \\
& Full Sample & Funds with $\beta>1$ \\
\hline$U N D E R(0 \%)_{i, m} \times\left(R_{t}^{B}-r_{t}\right)$ & 0.0315 & 0.0164 \\
& $(29.05)$ & $(16.53)$ \\
$U N D E R(5 \%)_{i, m} \times\left(R_{t}^{B}-r_{t}\right)$ & 0.0956 & 0.0970 \\
& $(21.31)$ & $(21.41)$ \\
$U N D E R(10 \%)_{i, m} \times\left(R_{t}^{B}-r_{t}\right)$ & 0.1564 & 0.1269 \\
& $(14.98)$ & $(13.82)$ \\
$U N D E R(15 \%)_{i, m} \times\left(R_{t}^{B}-r_{t}\right)$ & 0.1928 & 0.1725 \\
& $(7.58)$ & $(6.28)$ \\
$U N D E R(20 \%)_{i, m} \times\left(R_{t}^{B}-r_{t}\right)$ & 0.2328 & 0.2914 \\
Month fixed effects & $(5.03)$ & $(5.24)$ \\
Year fixed effects & Yes & Yes \\
$R^{2}$ & Yes & Yes \\
Wald Test & 0.7903 & 0.8112 \\
& & \\
N. of obs & 0.00 & 0.00 \\
\hline
\end{tabular}

Table 1 Regression of daily funds' excess returns on S\&P500 index's daily excess returns interacted with UNDER indicators. The fixed effects variables are interacted with $\left(R_{t}^{B}-r_{t}\right)$. Numbers in parenthesis correspond to t-statistics computed with robust and clustered standard errors. The row Wald Test displays p-values for the null hypothesis that the coefficients in the corresponding regression are jointly equal.

centives become more and more relevant as relative performance deteriorates (increasing coefficients of $U N D E R(b)_{i, m} \times\left(R_{t}^{B}-r_{t}\right)$ in the magnitude of the underperformance), leading underperforming managers to further increase the riskiness of their portfolio.

We complete our analysis by looking at the investment strategy of overperforming funds (Table 2). The analysis developed in Section 3 is not fully conclusive. As a matter of fact, in case of a collar type remuneration scheme we have that the investment strategy is monotonic and converges to the benchmark. Instead, in case of the other remuneration schemes we have that the optimal investment strategy converges to the one adopted in case of a linear remuneration scheme. The strategy foresees an investment in the risky asset in excess with respect to the benchmark if and only if $\mu-r-\beta \sigma^{2}>0$, otherwise the manager invests in the risky asset less than the benchmark. In the first case we expect a null exposure to the benchmark (decreasing in the overperformance of the fund), in the other cases the tracking error should be constant.

We have performed a regression analysis similar to the one developed for the case of underperforming funds. Analyzing the results exhibited in Table 2, we note a main difference between the two cases (full sample, funds with $\beta>1$ ) : for moderate levels of overperformance, the case of funds with beta higher than one shows positive coefficients that increase with the level of relative

Indeed, [Basak et al.(2007)] concentrate their analysis on funds with managers with a high risk aversion coefficient. 


\begin{tabular}{lcc}
\hline & \multicolumn{2}{c}{ Dependent Variable: $R_{i, t}^{F}-r_{t}$} \\
& Full Sample & Funds with $\beta>1$ \\
\hline OVER(0\%) $)_{i, m} \times\left(R_{t}^{B}-r_{t}\right)$ & -0.0343 & -0.0286 \\
OVER $(5 \%)_{i, m} \times\left(R_{t}^{B}-r_{t}\right)$ & $(-30.09)$ & $(-25.32)$ \\
& -0.1367 & 0.0547 \\
OVER $(10 \%)_{i, m} \times\left(R_{t}^{B}-r_{t}\right)$ & $(-16.39)$ & 0.0908 \\
& -0.1060 & $(2.72)$ \\
OVER $(15 \%)_{i, m} \times\left(R_{t}^{B}-r_{t}\right)$ & $(-2.93)$ & -0.0460 \\
OVER $(20 \%)_{i, m} \times\left(R_{t}^{B}-r_{t}\right)$ & -0.2604 & $(-0.71)$ \\
Month fixed effects & $(-2.95)$ & -0.2131 \\
Year fixed effects & -0.1011 & $(-2.87)$ \\
$R^{2}$ & $(-0.89)$ & Yes \\
Wald Test & Yes & 0.8109 \\
N. of obs & 0.7902 & 0.00 \\
\hline
\end{tabular}

Table 2 Regression of daily funds' excess returns on S\&P500 index's daily excess returns interacted with OVER indicators. The fixed effects variables are interacted with $\left(R_{t}^{B}-r_{t}\right)$. Numbers in parenthesis correspond to t-statistics computed with robust and clustered standard errors. The row Wald Test displays p-values for the null hypothesis that the coefficients in the corresponding regression are jointly equal.

performance. Then, as soon as overperformance is higher than $15 \%$, coefficients become negative. On the other hand, estimated coefficients are always negative when the full set of data is used. This seems to suggest that the managers risk attitude plays a relevant role in the definition of the risk incentives in case of overperformance with respect to the benchmark. Indeed, fund managers who are less risk averse tend to further increase their risk profile in case of moderate levels of overperformance. This gambling incentive, then, becomes weaker and weaker (till disappearing) as the performance further increases. These results agree with a collar type remuneration scheme and may suggest that the asset managers are capped in their remuneration yielding a lower exposure to the benchmark.

\section{Conclusions}

The debate on the misalignment between the behavior of the asset manager and the investors' interests is intense and non conclusive. The main issue concerns the incentives to take excessive risk by the manager. The debate mainly regards two features: asset- vs. performance-based remuneration schemes, asymmetric vs. fulcrum type fees.

There is a well established claim that asymmetric fees based on the performance induce the asset manager to take risk in excess whereas a fulcrum type fee should prevent it. On the other hand, asset-based fees should perform bet- 
ter than performance-based fees in preventing excessive risk taking but may not be efficient in aligning interests of asset managers and of investors.

Concentrating our attention on remuneration schemes related to the performance of the fund with respect to a benchmark, we have shown that it is not the asymmetric-fulcrum type feature that makes the difference in preventing excessive risk taking in case of a poor performance. To prevent gambling when the performance deteriorates, it is important that the asset manager is not endowed with a fixed fee and that his/her remuneration is sensitive to a very poor relative performance.

\section{A Proofs}

To prove the theorems, let us consider an economy with zero risk-free rate $\left(r_{*}=0\right)$, a risky asset with drift $\mu_{*}=\mu-r-\beta \sigma^{2}$ and volatility $\sigma$. Therefore the evolution of the relative performance process $X(t)$ in (2) is fully described by

$$
\begin{aligned}
d X(t) & =X(t)\left[\delta(t) \mu_{*} d t+\delta(t) \sigma d Z(t)\right] \\
& =X(t)\left[\left(r_{*}+\delta(t)\left(\mu_{*}-r_{*}\right)\right) d t+\delta(t) \sigma d Z(t)\right],
\end{aligned}
$$

and thus in this new economy $X$ is the process which describes the portfolio value (if the agent can invest in both the risky and the risk-free asset) and $\delta$ is the amount of wealth (in percentage) invested in the risky asset.

In this framework the state price density is given by

$$
\xi(t)=e^{-\left(r_{*}+|\vartheta|^{2} / 2\right) t-\vartheta Z(t)}=e^{-|\vartheta|^{2} / 2 t-\vartheta Z(t)}
$$

with $\vartheta=\frac{\mu_{*}-r_{*}}{\sigma}=\frac{\mu-r-\sigma^{2} \beta}{\sigma}$.

The outline of all the proofs is the following, see [Karatzas and Shreve(1998)] for details: once an extended concave utility function $\widetilde{U}$ is defined, we compute its set-valued first order derivative $\widetilde{U}^{\prime}$ as well as the function $I$, which is the inverse of $\widetilde{U}^{\prime}$ in the sense that $z \in \widetilde{U}^{\prime}(I(z))$

The starting problem is equivalent to the following:

$$
\max _{X(T)} E[\widetilde{U}(X(T))] \text { such that } E[\xi(T) X(T)] \leq X(0) \text { and } X(T) \geq 0 .
$$

Therefore the martingale approach considered, for example, in [Carpenter(2000)] can be extended to solve our problems, with $X^{*}(T)=I(\lambda \xi(T))$ ), and with $\lambda$, the Lagrangian multiplier, solution of

$$
E[\xi(T) I(\lambda \xi(T))]=X(0)=1 .
$$

We would like to stress that the function $\Xi(\lambda)=E[\xi(T) I(\lambda \xi(T))]$ is continuous and strictly decreasing. Furthermore $\lim _{\lambda \rightarrow+\infty} \Xi(\lambda)=0$ and $\lim _{\lambda \rightarrow 0} \Xi(\lambda)=+\infty$. Therefore a solution of Equation (14) always exists.

The optimal process $X^{*}$ is then computed for any $t \in[0, T]$ as

$$
\left.X^{*}(t)=E_{t}\left[\frac{\xi(T)}{\xi(t)} X^{*}(T)\right]=E_{t}\left[\frac{\xi(T)}{\xi(t)} I(\lambda \xi(T))\right)\right] .
$$

Notice that the process $X^{*}$ is the optimal process also for the initial problem (with nonconcave utility function $U$ ) due to [Carpenter(2000), Equations (A5)-(A8)]. 
To deal with the optimal allocation process, let us now define $M(t)=\xi(t) X^{*}(t)$; being $M$ a martingale [Karatzas and Shreve(1998)], there exists a function $\varphi$ such that $d M(t)=$ $\varphi(t) d Z(t)$. Once $\varphi$ is computed, due to [Karatzas and Shreve(1998), Theorem 3.7.3], we have

$$
\delta^{*}=\frac{1}{\sigma}\left(\vartheta+\frac{\varphi(t)}{M(t)}\right)
$$

In the following, we also denote with $i$ is the inverse of the first order derivative of $u$, i.e. $i(z)=\left(u^{\prime}\right)^{-1}(z)=z^{1 /(a-1)}$.

\section{A.1 Proof of Theorem 1}

Let us define the extended utility function

$$
\widetilde{U}(x)=\left\{\begin{array}{l}
U(x):=(m x+K)^{a} / a \\
-\infty \quad \text { if } x<0
\end{array} \text { if } x \geq 0,\right.
$$

If $m=1$ and $K=0$ we obtain the classical Merton problem with terminal utility. The utility function $\widetilde{U}$ is not differentiable in 0 , therefore we can define a set-valued function $\widetilde{U}^{\prime}:[0,+\infty) \rightarrow(0,+\infty)$ by

$$
\widetilde{U}^{\prime}(x)=\left\{\begin{array}{l}
\left\{U^{\prime}(x)\right\} \quad \text { if } x>0 \\
{\left[U^{\prime}(0),+\infty\right) \quad \text { if } x=0}
\end{array}\right.
$$

and its inverse

$$
I(z)=\frac{1}{m}\left(i\left(\frac{z}{m}\right)-K\right) \mathbf{1}_{z<U^{\prime}(0)} .
$$

Equation (15) implies

$$
X^{*}(t)=-\frac{K}{m} E_{t}\left[\frac{\xi(T)}{\xi(t)} \mathbf{1}_{\xi(T)<\gamma}\right]+\frac{1}{m} E_{t}\left[\frac{\xi(T)}{\xi(t)} i\left(\frac{\lambda \xi(T)}{m}\right) \mathbf{1}_{\xi(T)<\gamma}\right]
$$

with $\gamma=U^{\prime}(0) / \lambda$, i.e. Equation (4), since

$$
E_{t}\left[\frac{\xi(T)}{\xi(t)} \mathbf{1}_{\xi(T)<\gamma}\right]=N\left(d_{1}(t)\right)
$$

with $d_{1}(t)=\left(\ln (\gamma / \xi(t))-|\vartheta|^{2}(T-t) / 2\right) /(|\vartheta| \sqrt{T-t})$, and

$$
E_{t}\left[\frac{\xi(T)}{\xi(t)} i\left(\frac{\lambda \xi(T)}{m}\right) \mathbf{1}_{\xi(T)<\gamma}\right]=K \frac{N^{\prime}\left(d_{1}(t)\right)}{N^{\prime}\left(d_{2}(t)\right)} N\left(d_{2}(t)\right)
$$

with $d_{2}(t)=d_{1}(t)+|\vartheta| \sqrt{T-t} /(1-a)$.

Finally, computations lead to

$$
\varphi(t)=\vartheta \xi(t)\left(\frac{a}{1-a} X^{*}(t)+\frac{K}{m} \frac{N\left(d_{1}(t)\right)}{1-a}\right)
$$

and therefore Equation (16) gives Equation (3). 


\section{A.2 Proof of Theorem 2}

This result follows directly from [Carpenter(2000)]. More precisely, (6) and (7) correspond to [Carpenter(2000), Equation (26)] and [Carpenter(2000), Equation (25)], respectively. More precisely, in [Carpenter(2000)] the author solves the optimization problem

$$
\max _{X(T)} E\left[u\left(\alpha\left(X(T)-B_{0} e^{r_{*} T}\right)^{+}+K\right)\right]
$$

which corresponds to our problem (in the new economy) setting $\alpha=1$, and $B_{0}=1$. Notice that in [Carpenter(2000)] $K$ should be positive: however the results can be easily extended to the case $K=0$, assuming $a \in(0,1)$.

More precisely, since the utility function $U(x):=u\left((x-1)^{+}+K\right)$ is not concave in $x$, we define the new concavified utility function

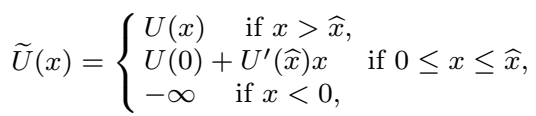

Let us consider the case $K=0$ : in order to have a continuous concavified utility function $\widetilde{U}$ it must be $\widehat{x}=1 /(1-a)$, which is greater than 1 since $a \in(0,1)$ (moreover, if $K=0$ and $a$ is negative, $U(0)=-\infty)$. For the case $K>0$ a similar result holds true with the existence (and uniqueness) of $\widehat{x}>1$ such that $U(\widehat{x})=U(0)+U^{\prime}(\widehat{x}) \widehat{x}$, thanks to [Carpenter(2000), Lemma 1].

The concavified utility function $\widetilde{U}$ is not differentiable in 0 , therefore we can define a set-valued function $\widetilde{U}^{\prime}:[0,+\infty) \rightarrow(0,+\infty)$ by

$$
\widetilde{U}^{\prime}(x)= \begin{cases}\left\{U^{\prime}(x)\right\} & \text { if } x>\widehat{x}, \\ \left\{U^{\prime}(\widehat{x})\right\} & \text { if } 0<x \leq \widehat{x}, \\ {\left[U^{\prime}(\widehat{x}),+\infty\right) \quad \text { if } x=0 .}\end{cases}
$$

See [Carpenter(2000), Appendix]. We can also define the inverse for the function $\widetilde{U}^{\prime}$ given by

$$
I(z)=(i(z)+1-K) \mathbf{1}_{z<U^{\prime}(\widehat{x})}
$$

where $I$ is necessary to compute $\lambda$ exploiting Equation (14), and thus to get the coefficients in (6) and (7)

$$
\begin{aligned}
& d_{1}(t)=\left(\ln (\gamma / \xi(t))-|\vartheta|^{2}(T-t) / 2\right) /(|\vartheta| \sqrt{T-t}), \\
& d_{2}(t)=d_{1}(t)+|\vartheta| \sqrt{T-t} /(1-a),
\end{aligned}
$$

being $\gamma=u^{\prime}(\widehat{x}-1+K) / \lambda$.

\section{A.3 Proof of Theorem 3}

Since the utility function $U(x):=u\left((x-1)^{+}-(x-\mathcal{H})^{+}+K\right)$ is not concave in $x$, we compute its concavification as in Equation (17). The concavified utility function $\widetilde{U}$ is not differentiable in 0 and $\mathcal{H}$, therefore we can define a set-valued function $\widetilde{U}^{\prime}:[0,+\infty) \rightarrow(0,+\infty)$ by

$$
\widetilde{U}^{\prime}(x)=\left\{\begin{array}{l}
{\left[0, U^{\prime}\left(\mathcal{H}^{-}\right)\right) \quad \text { if } x \geq \mathcal{H},} \\
\left\{U^{\prime}(x)\right\} \quad \text { if } \widehat{x}<x<\mathcal{H}, \\
\left\{U^{\prime}(\widehat{x})\right\} \quad \text { if } 0<x \leq \widehat{x}, \\
{\left[U^{\prime}(\widehat{x}),+\infty\right) \quad \text { if } x=0,}
\end{array}\right.
$$

where $\widehat{x}$ is as in A.2. We can also define an inverse for the function $\widetilde{U}^{\prime}$ given by

$$
I(z)=(i(z)+1-K) \mathbf{1}_{U^{\prime}\left(\mathcal{H}^{-}\right) \leq z<U^{\prime}(\widehat{x})}+\mathcal{H} \mathbf{1}_{z<U^{\prime}\left(\mathcal{H}^{-}\right)} .
$$


Let us define $\gamma_{1}=U^{\prime}\left(\mathcal{H}^{-}\right) / \lambda$, and $\gamma_{2}=U^{\prime}(\widehat{x}) / \lambda$, Equation (15) implies

$$
\begin{aligned}
X^{*}(t)= & \mathcal{H} E_{t}\left[\frac{\xi(T)}{\xi(t)} \mathbf{1}_{\xi(T)<\gamma_{1}}\right]+(1-K) E_{t}\left[\frac{\xi(T)}{\xi(t)} \mathbf{1}_{\gamma_{1} \leq \xi(T)<\gamma_{2}}\right] \\
& +E_{t}\left[i(\lambda \xi(T)) \frac{\xi(T)}{\xi(t)} \mathbf{1}_{\gamma_{1} \leq \xi(T)<\gamma_{2}}\right],
\end{aligned}
$$

i.e. Equation (9) with

$$
\begin{aligned}
& d_{1}(t)=\left(\ln \left(\gamma_{1} / \xi(t)\right)-|\vartheta|^{2}(T-t) / 2\right) /(|\vartheta| \sqrt{T-t}), \\
& d_{2}(t)=d_{1}(t)+|\vartheta| \sqrt{T-t} /(1-a), \\
& d_{3}(t)=\left(\ln \left(\gamma_{2} / \xi(t)\right)-|\vartheta|^{2}(T-t) / 2\right) /(|\vartheta| \sqrt{T-t}), \\
& d_{4}(t)=d_{3}(t)+|\vartheta| \sqrt{T-t} /(1-a) .
\end{aligned}
$$

since

$$
\begin{aligned}
& E_{t}\left[\frac{\xi(T)}{\xi(t)} \mathbf{1}_{\xi(T)<\gamma_{1}}\right]=N\left(d_{1}(t)\right), \quad E_{t}\left[\frac{\xi(T)}{\xi(t)} \mathbf{1}_{\xi(T)<\gamma_{2}}\right]=N\left(d_{3}(t)\right), \\
& E_{t}\left[\frac{\xi(T)}{\xi(t)} i\left(\frac{\lambda \xi(T)}{1+m}\right) \mathbf{1}_{\xi(T)<\gamma_{1}}\right]=(\mathcal{H}+K-1) \frac{N^{\prime}\left(d_{1}(t)\right)}{N^{\prime}\left(d_{2}(t)\right)} N\left(d_{2}(t)\right), \\
& E_{t}\left[\frac{\xi(T)}{\xi(t)} i\left(\frac{\lambda \xi(T)}{1+m}\right) \mathbf{1}_{\xi(T)<\gamma_{2}}\right]=(\widehat{x}+K-1) \frac{N^{\prime}\left(d_{3}(t)\right)}{N^{\prime}\left(d_{4}(t)\right)} N\left(d_{4}(t)\right) .
\end{aligned}
$$

The optimal strategy $\delta^{*}$ is computed as in A.1, exploiting Equation (16).

\section{A.4 Proof of Theorem 4}

In this case $U(x):=u\left((x-1)^{+}-(x-\mathcal{H})^{+}+m x+K\right)$ and the concavified utility function

$$
\tilde{U}(x)=\left\{\begin{array}{l}
U(x) \quad \text { if } x>\widehat{x}_{u}, \\
U(0)+U^{\prime}\left(\widehat{x}_{u}\right) x \\
-\infty \quad \text { if } x<0
\end{array} \text { if } 0 \leq x \leq \widehat{x}_{u}\right.
$$

is not differentiable in 0 and $\mathcal{H}$, therefore we can define a set-valued function $\widetilde{U}^{\prime}$ as

$$
\widetilde{U}^{\prime}(x)=\left\{\begin{array}{l}
\left\{U^{\prime}(x)\right\} \quad \text { if } x>\mathcal{H}, \\
{\left[U^{\prime}\left(\mathcal{H}^{+}\right), U^{\prime}\left(\mathcal{H}^{-}\right)\right] \quad \text { if } x=\mathcal{H}} \\
\left\{U^{\prime}(x)\right\} \quad \text { if } \widehat{x}_{u}<x<\mathcal{H}, \\
\left\{U^{\prime}\left(\widehat{x}_{u}\right)\right\} \quad \text { if } 0<x \leq \widehat{x}_{u}, \\
{\left[U^{\prime}\left(\widehat{x}_{u}\right),+\infty\right) \quad \text { if } x=0 .}
\end{array}\right.
$$

We can also define an inverse for the function $\widetilde{U}^{\prime}$ given by

$$
\begin{aligned}
I(z) & =\frac{1}{1+m}\left(i\left(\frac{z}{1+m}\right)+1-K\right) \mathbf{1}_{U^{\prime}\left(\mathcal{H}^{-}\right)<z<U^{\prime}\left(\widehat{x}_{u}\right)}+\mathcal{H} \mathbf{1}_{U^{\prime}\left(\mathcal{H}^{+}\right) \leq z \leq U^{\prime}\left(\mathcal{H}^{-}\right)} \\
& +\frac{1}{m}\left(i\left(\frac{z}{m}\right)+1-K-\mathcal{H}\right) \mathbf{1}_{z<U^{\prime}\left(\mathcal{H}^{+}\right)} .
\end{aligned}
$$

Let us define $\gamma_{1}=U^{\prime}\left(\mathcal{H}^{+}\right) / \lambda, \gamma_{2}=U^{\prime}\left(\mathcal{H}^{-}\right) / \lambda$, and $\gamma_{3}=U^{\prime}\left(\widehat{x}_{u}\right) / \lambda$, then

$$
\begin{aligned}
X^{*}(t) & =\frac{1}{1+m} E_{t}\left[\frac{\xi(T)}{\xi(t)} i\left(\frac{\lambda \xi(T)}{1+m}\right) \mathbf{1}_{\gamma_{2}<\xi(T)<\gamma_{3}}\right]+\frac{1-K}{1+m} E_{t}\left[\frac{\xi(T)}{\xi(t)} \mathbf{1}_{\gamma_{2}<\xi(T)<\gamma_{3}}\right] \\
& +\mathcal{H} E_{t}\left[\frac{\xi(T)}{\xi(t)} \mathbf{1}_{\gamma_{1}<\xi(T)<\gamma_{2}}\right]+\frac{1}{m} E_{t}\left[\frac{\xi(T)}{\xi(t)} i\left(\frac{\lambda \xi(T)}{m}\right) \mathbf{1}_{\xi(T)<\gamma_{1}}\right] \\
& +\frac{1-K-\mathcal{H}}{m} E_{t}\left[\frac{\xi(T)}{\xi(t)} \mathbf{1}_{\xi(T)<\gamma_{1}}\right],
\end{aligned}
$$


i.e. Equation (10) with

$$
\begin{aligned}
& d_{1}(t)=\left(\ln \left(\gamma_{1} / \xi(t)\right)-|\vartheta|^{2}(T-t) / 2\right) /(|\vartheta| \sqrt{T-t}), \\
& d_{2}(t)=d_{1}(t)+|\vartheta| \sqrt{T-t} /(1-a), \\
& d_{3}(t)=\left(\ln \left(\gamma_{2} / \xi(t)\right)-|\vartheta|^{2}(T-t) / 2\right) /(|\vartheta| \sqrt{T-t}), \\
& d_{4}(t)=d_{3}(t)+|\vartheta| \sqrt{T-t} /(1-a), \\
& d_{5}(t)=\left(\ln \left(\gamma_{3} / \xi(t)\right)-|\vartheta|^{2}(T-t) / 2\right) /(|\vartheta| \sqrt{T-t}), \\
& d_{6}(t)=d_{5}(t)+|\vartheta| \sqrt{T-t} /(1-a) .
\end{aligned}
$$

The optimal strategy $\delta^{*}$ is computed as in A.1, exploiting Equation (16).

\section{A.5 Proof of Theorem 5}

In this case $U(x):=u\left((x-1)^{+}-(x-\mathcal{H})^{+}+m x+K\right)$ and the concavified utility function is given by

$$
\widetilde{U}(x)=\left\{\begin{array}{l}
U(x) \quad \text { if } 0 \leq x<\widehat{x}_{d} \text { or } x>\widehat{x}_{u}, \\
U\left(x_{d}\right)+U^{\prime}\left(\widehat{x}_{u}\right)\left(x-x_{d}\right) \quad \text { if } \widehat{x}_{d} \leq x \leq \widehat{x}_{u}, \\
-\infty \quad \text { if } x<0 ;
\end{array}\right.
$$

since $\widetilde{U}$ is not differentiable in 0 and $\mathcal{H}$, we can define a set-valued function $\widetilde{U}^{\prime}$ as

$$
\widetilde{U}^{\prime}(x)=\left\{\begin{array}{l}
\left\{U^{\prime}(x)\right\} \quad \text { if } x>\mathcal{H}, \quad \widehat{x}_{u}<x<\mathcal{H}, \text { or } 0<x<\widehat{x}_{d} \\
{\left[U^{\prime}(\mathcal{H}+), U^{\prime}\left(\mathcal{H}^{-}\right)\right] \text {if } x=\mathcal{H},} \\
\left\{U^{\prime}\left(\widehat{x}_{u}\right)\right\} \text { if } \widehat{x}_{d} \leq x \leq \widehat{x}_{u} \\
{\left[U^{\prime}\left(\widehat{x}_{u}\right),+\infty\right) \text { if } x=0}
\end{array}\right.
$$

The inverse for the function $\widetilde{U}^{\prime}$ is given by

$$
\begin{aligned}
I(z) & =\frac{1}{m}\left(i\left(\frac{z}{m}\right)-K\right) \mathbf{1}_{U^{\prime}\left(\widehat{x}_{u}\right)<z<U^{\prime}(0)}+\widehat{x}_{u} \mathbf{1}_{z=U^{\prime}\left(\widehat{x}_{u}\right)} \\
& +\frac{1}{1+m}\left(i\left(\frac{z}{1+m}\right)+1-K\right) \mathbf{1}_{U^{\prime}\left(\mathcal{H}^{-}\right)<z<U^{\prime}\left(\widehat{x}_{u}\right)}+\mathcal{H} \mathbf{1}_{U^{\prime}\left(\mathcal{H}^{+}\right) \leq z \leq U^{\prime}\left(\mathcal{H}^{-}\right)} \\
& +\frac{1}{m}\left(i\left(\frac{z}{m}\right)+1-K-\mathcal{H}\right) \mathbf{1}_{z<U^{\prime}\left(\mathcal{H}^{+}\right)} .
\end{aligned}
$$

Let us define $\gamma_{1}=U^{\prime}\left(\mathcal{H}^{+}\right) / \lambda, \gamma_{2}=U^{\prime}\left(\mathcal{H}^{-}\right) / \lambda, \gamma_{3}=U^{\prime}\left(\widehat{x}_{u}\right) / \lambda$, and $\gamma_{4}=U^{\prime}(0) / \lambda$, then

$$
\begin{aligned}
X^{*}(t) & =\frac{1}{m} E_{t}\left[\frac{\xi(T)}{\xi(t)} i\left(\frac{\lambda \xi(T)}{m}\right) \mathbf{1}_{\gamma_{3}<\xi(T)<\gamma_{4}}\right]-\frac{K}{m} E_{t}\left[\frac{\xi(T)}{\xi(t)} \mathbf{1}_{\gamma_{3}<\xi(T)<\gamma_{4}}\right] \\
& +\frac{1}{1+m} E_{t}\left[\frac{\xi(T)}{\xi(t)} i\left(\frac{\lambda \xi(T)}{1+m}\right) \mathbf{1}_{\gamma_{2}<\xi(T)<\gamma_{3}}\right]+\frac{1-K}{1+m} E_{t}\left[\frac{\xi(T)}{\xi(t)} \mathbf{1}_{\gamma_{2}<\xi(T)<\gamma_{3}}\right] \\
& +\mathcal{H} E_{t}\left[\frac{\xi(T)}{\xi(t)} \mathbf{1}_{\gamma_{1}<\xi(T)<\gamma_{2}}\right]+\frac{1}{m} E_{t}\left[\frac{\xi(T)}{\xi(t)} i\left(\frac{\lambda \xi(T)}{m}\right) \mathbf{1}_{\xi(T)<\gamma_{1}}\right] \\
& +\frac{1-K-\mathcal{H}}{m} E_{t}\left[\frac{\xi(T)}{\xi(t)} \mathbf{1}_{\xi(T)<\gamma_{1}}\right]
\end{aligned}
$$


i.e. Equation (11) with

$$
\begin{aligned}
& d_{1}(t)=\left(\ln \left(\gamma_{1} / \xi(t)\right)-|\vartheta|^{2}(T-t) / 2\right) /(|\vartheta| \sqrt{T-t}), \\
& d_{2}(t)=d_{1}(t)+|\vartheta| \sqrt{T-t} /(1-a), \\
& d_{3}(t)=\left(\ln \left(\gamma_{2} / \xi(t)\right)-|\vartheta|^{2}(T-t) / 2\right) /(|\vartheta| \sqrt{T-t}), \\
& d_{4}(t)=d_{3}(t)+|\vartheta| \sqrt{T-t} /(1-a), \\
& d_{5}(t)=\left(\ln \left(\gamma_{3} / \xi(t)\right)-|\vartheta|^{2}(T-t) / 2\right) /(|\vartheta| \sqrt{T-t}), \\
& d_{6}(t)=d_{5}(t)+|\vartheta| \sqrt{T-t} /(1-a), \\
& d_{7}(t)=\left(\ln \left(\gamma_{4} / \xi(t)\right)-|\vartheta|^{2}(T-t) / 2\right) /(|\vartheta| \sqrt{T-t}), \\
& d_{8}(t)=d_{7}(t)+|\vartheta| \sqrt{T-t} /(1-a) .
\end{aligned}
$$

The optimal strategy $\delta^{*}$ is computed as in A.1, exploiting Equation (16).

\section{A.6 Proof of Theorem 6}

In this case $U(x):=u\left(p\left(x-H_{1}\right)^{+}+m x-c\left(H_{2}-x\right)^{+}+K\right)$ and the concavified utility function is defined as in (18) and is not differentiable in 0 , therefore we define its first order derivative as

$$
\widetilde{U}^{\prime}(x)=\left\{\begin{array}{l}
\left\{U^{\prime}(x)\right\} \quad \text { if } x>\widehat{x}_{u}, \\
\left\{U^{\prime}\left(\widehat{x}_{u}\right)\right\} \quad \text { if } 0<x \leq \widehat{x}_{u}, \\
{\left[U^{\prime}\left(\widehat{x}_{u}\right),+\infty\right) \quad \text { if } x=0 .}
\end{array}\right.
$$

Its inverse is given by

$$
I(z)=\frac{1}{m+p}\left(i\left(\frac{z}{m+p}\right)-K+p H_{1}\right) \mathbf{1}_{z<U^{\prime}\left(\widehat{x}_{u}\right)} .
$$

Therefore

$$
X^{*}(t)=\frac{p H_{1}-K}{m+p} E_{t}\left[\frac{\xi(T)}{\xi(t)} \mathbf{1}_{\xi(T)<\gamma}\right]+\frac{1}{m+p} E_{t}\left[\frac{\xi(T)}{\xi(t)} i\left(\frac{\lambda \xi(T)}{m+p}\right) \mathbf{1}_{\xi(T)<\gamma}\right]
$$

with $\gamma=U^{\prime}\left(\widehat{x}_{u}\right) / \lambda$, i.e. Equation (12) with

$$
\begin{aligned}
& d_{1}(t)=\left(\ln (\gamma / \xi(t))-|\vartheta|^{2}(T-t) / 2\right) /(|\vartheta| \sqrt{T-t}), \\
& d_{2}(t)=d_{1}(t)+|\vartheta| \sqrt{T-t} /(1-a) .
\end{aligned}
$$

The optimal strategy $\delta^{*}$ is computed as in A.1, exploiting Equation (16).

\section{A.7 Proof of Theorem 7}

In this case $U(x):=u\left(p\left(x-H_{1}\right)^{+}+m x-c\left(H_{2}-x\right)^{+}+K\right)$ and the concavified utility function is defined as in (19) and is not differentiable in 0 , therefore we define its first order derivative

$$
\widetilde{U}^{\prime}(x)=\left\{\begin{array}{l}
\left\{U^{\prime}(x)\right\} \quad \text { if } 0<x<\widehat{x}_{d} \text { or } x>\widehat{x}_{u}, \\
\left\{U^{\prime}\left(\widehat{x}_{u}\right)\right\} \quad \text { if } \widehat{x}_{d} \leq x \leq \widehat{x}_{u} \\
{\left[U^{\prime}(0),+\infty\right) \quad \text { if } x=0}
\end{array}\right.
$$


In this case the inverse for the function $\widetilde{U}^{\prime}$ is given by

$$
\begin{aligned}
I(z) & =\frac{1}{m+p}\left(i\left(\frac{z}{m+p}\right)+p H_{1}-K\right) \mathbf{1}_{z<U^{\prime}\left(\widehat{x}_{u}\right)}+\widehat{x}_{u} \mathbf{1}_{z=U^{\prime}\left(\widehat{x}_{u}\right)} \\
& +\frac{1}{m+c}\left(i\left(\frac{z}{m+c}\right)+c H_{2}-K\right) \mathbf{1}_{U^{\prime}\left(\widehat{x}_{u}\right)<z<U^{\prime}(0)} .
\end{aligned}
$$

Therefore we have

$$
\begin{aligned}
X^{*}(t) & =\frac{1}{m+p} E_{t}\left[\frac{\xi(T)}{\xi(t)} i\left(\frac{\lambda \xi(T)}{m+p}\right) \mathbf{1}_{\xi(T)<\gamma_{1}}\right]+\frac{p H_{1}-K}{m+p} E_{t}\left[\frac{\xi(T)}{\xi(t)} \mathbf{1}_{\xi(T)<\gamma_{1}}\right] \\
& +\frac{1}{m+c} E_{t}\left[\frac{\xi(T)}{\xi(t)} i\left(\frac{\lambda \xi(T)}{m+c}\right) \mathbf{1}_{\gamma_{1}<\xi(T)<\gamma_{2}}\right]+\frac{c H_{2}-K}{m+c} E_{t}\left[\frac{\xi(T)}{\xi(t)} \mathbf{1}_{\gamma_{1}<\xi(T)<\gamma_{2}}\right],
\end{aligned}
$$

with $\gamma_{1}=U^{\prime}\left(\widehat{x}_{u}\right) / \lambda=U^{\prime}\left(\widehat{x}_{d}\right) / \lambda$, and $\gamma_{2}=U^{\prime}(0) / \lambda$, i.e. Equation (13) with

$$
\begin{aligned}
& d_{1}(t)=\left(\ln \left(\gamma_{1} / \xi(t)\right)-|\vartheta|^{2}(T-t) / 2\right) /(|\vartheta| \sqrt{T-t}), \\
& d_{2}(t)=d_{1}(t)+|\vartheta| \sqrt{T-t} /(1-a), \\
& d_{3}(t)=\left(\ln \left(\gamma_{2} / \xi(t)\right)-|\vartheta|^{2}(T-t) / 2\right) /(|\vartheta| \sqrt{T-t}), \\
& d_{4}(t)=d_{3}(t)+|\vartheta| \sqrt{T-t} /(1-a) .
\end{aligned}
$$

The optimal strategy $\delta^{*}$ is computed as in A.1, exploiting Equation (16).

\section{References}

[Admati and Pfledeirer(1997)] Admati, A., Pfleiderer, P.: Does it all add up? Benchmarks and the compensation of active portfolio managers. J. Bus. 70, 323-350 (1997)

[Aragon and Nanda(2014)] Aragon, G., Nanda, V.: Opaque trading, disclosure, and asset prices: implications for hedge fund regulation. Rev. Financial Stud. 27, 1190-1237 (2014)

[Basak et al.(2007)] Basak, S., Pavlova, A., Shapiro, A.: Optimal asset allocation and risk shifting in money management. Rev. Financial Stud. 20, 1583-1621 (2007)

[Basak et al.(2008)] Basak, S., Pavlova, A., Shapiro, A.: Offsetting the implicit incentives: benefits of benchmarking in money management. J. Bank. Finance 32, 1883-1893 (2008)

[Brown et al.(1996)] Brown, K., Harlow, W., Starks, L.: Of tournaments and temptations: an analysis of managerial incentives in the mutual fund industry. J. Finance 51, 85-110 (1996)

[Buraschi et al.(2014)] Buraschi, A., Kosowski, R., Sritrakul, W.: Incentives and endogenous risk taking: a structural view on hedge fund alphas. J. Finance 69, 2819-2869 (2014)

[Busse(2001)] Busse, J.: Another look at mutual fund tournaments. J. Financial Quant. Anal. 36, 53-73 (2001)

[Carpenter(2000)] Carpenter, J.: Does option compensation increase managerial risk appetite? J. Finance LV, 2311-2331 (2000)

[Chen and Pennacchi(2009)] Chen, H., Pennacchi, G.: Does prior performance affect a mutual fund's choice of risk? Theory and further empirical evidence. J. Financial Quant. Anal. 44, 745-775 (2009)

[Chevalier and Ellison(1997)] Chevalier, J., Ellison, G.: Risk taking by mutual funds as a response to incentives. J. Political Econ. 105, 1167-1200 (1997)

[Christoffersen and Simutin(2016)] Christoffersen, S.K., Simutin, M.: On the demand for high-beta stocks: Evidence from mutual funds. SSRN 2022266 (2016)

[Cuoco and Kaniel(2011)] Cuoco, D., Kaniel, R.: Equilibrium prices in the presence of delegated portfolio management. J. Financial Econ. 101, 264-296 (2011)

[Dai and Sundaresan(2009)] Dai, Q., Sundaresan, S.: Risk management framework for hedge funds: role of funding and redemption options on leverage. SSRN 1439706 (2009) 
[Das and Sundaram(2002)] Das, S., Sundaram, R.: Fee speech: signaling, risk-sharing and the impact of fee structures on investor welfare. Rev. Financial Stud. 15(5), 1465-1497 (2002)

[Golec(2003)] Golec, J.: Regulation and the rise in asset-based mutual fund management fees. J. Financial Res. 26, 19-30 (2003)

[Golec and Starks(2004)] Golec, J., Starks, L.: Performance fee contract and mutual fund risk. J. Financial Econ. 73, 93-118 (2004)

[Gordon and Baptista(2010)] Gordon, J.A., Baptista, A.M.: Active portfolio management with benchmarking: a frontier based on alpha. J. Bank. Finance 34, 2185-2197 (2010)

[Grinblatt and Titman(1989)] Grinblatt, M., Titman, S.: Adverse risk incentives and the design of performance-based contracts. Manag. Sci. 35, 807-822 (1989)

[Hodder and Jackwerth(2007)] Hodder, J., Jackwerth, J.C.: Incentive contracts and hedge fund management. J. Financial Quant. Anal. 42, 811-826 (2007)

[Karatzas and Shreve(1998)] Karatzas, I., Shreve, S.: Methods of Mathematical Finance. Springer, New York (1998)

[Koijen(2014)] Koijen, R., 2014: The cross-section of managerial ability, incentives and risk preferences. J. Finance 69, 1051-1098 (2014)

[Lewellen and Nagel(2006)] Lewellen, J., Nagel, S.: The conditional CAPM does not explain asset-pricing anomalies. J. Financial Econ. 82, 289-314 (2006)

[Lynch and Musto(1997)] Lynch, A., Musto, D.: Understanding fee structures in the asset management business. SSRN 1297754 (1997)

[Massa and Patgiri(2009)] Massa, M., Patgiri, R.: Incentives and mutual fund performance: higher performance or just higher risk taking? Rev. Financial Stud. 22, 1777-1815 (2009)

[Nicolosi et al.(2017)] Nicolosi, M., Angelini, F., Herzel, S.: Portfolio management with benchmark related incentives under mean reverting processes. Annals Operations Res., Online First (2017)

[Ou-Yang(2003)] Ou-Yang, H.: Optimal contracts in a continuous-time delegated portfolio management problem. Rev. Financial Stud. 16, 173-208 (2003)

[Ross(2004)] Ross, S.: Compensation, incentives, and the duality of risk aversion and riskiness. J. Finance 59, 207-225 (2004)

[Sirri and Tufano(1998)] Sirri, E., Tufano, P.: Costly search and mutual fund flows. J. Finance 54, 359-375 (1998) 